\title{
Lerncoaching an der Fachhochschule Südwestfalen in Meschede
}

\section{Individuelle Unterstützungen für eine heterogene Studierendenschaft}

\author{
An der Fachhochschule Südwestfalen hat das Thema Lerncoaching seit \\ Jahren einen festen Platz. Der Autor, Studierendencoach am Standort \\ Meschede, berichtet über das Angebot an lernunterstützenden Maßnahmen \\ und etablierten Programmen.
}

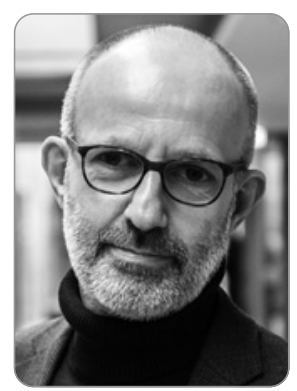

\section{Dr. Torsten Pätzold}

war als Lehrbeauftragter für verschiedene Bildungseinrichtungen und Fachhochschulen vor allem in den Bereichen Lern- und Arbeitsmethodik, Selbst- und Zeitmanagement und weitere Methodenkompetenzen tätig. Er absolvierte eine Weiterbildung in der personenzentrierten Beratung nach Carl Rogers bei der Gesellschaft für Gesprächspsychotherapie, ist zertifizierter Entspannungspädagoge und NLP-Master-Coach. Dr. Torsten Pätzold ist seit 2007 für die Studienberatung und das Studierendencoaching am Standort Meschede der Fachhochschule Südwestfalen verantwortlich. Seinen Schwerpunkt bildet das Lerncoaching sowie die Planung und Koordination von individuellen Studienverläufen.

Stichwörter: Lerncoaching, Lernen, Studienanfänger, Lernprobleme, Prüfungsstress

Die Fachhochschule Südwestfalen ist eine Flächenhochschule mit Standorten in Iserlohn, Hagen, Soest und Meschede. An der Hochschule studieren insgesamt ca. 14.000, in Meschede ca. 2.000 junge Menschen in aktuell acht Bachelorund drei Masterstudiengängen. Das Studienangebot in Meschede ist in die drei Lehreinheiten Elektrotechnik, Maschinenbau und Wirtschaft strukturiert. Aus historischen Gründen bilden diese drei Lehreinheiten einen einzigen Fachbereich. Dieser Umstand erleichtert die Kommunikation zwischen allen Akteuren erheblich.

Naturgemäß ist die Studierendenschaft an einer Fachhochschule heterogener, als das allgemein auf Universitäten zutrifft. Neben den klassischen Abiturienten finden junge Menschen mit einer Fachhochschulreife, Meister/-innen, Techniker/-innen sowie beruflich qualifizierte Studienanfänger/-innen den Weg in die Fachhochschule. Der Teil der sogenannten Bildungsaufsteiger, also der Studierenden, die nicht aus Akademikerfamilien stammen, ist an Fach- hochschulen hoch. Da seit einigen Jahren die Landespolitik in NRW die Anzahl der Hochschulabsolventen signifikant erhöhen möchte, hat das einen Einfluss auf die gesamte Bildungslandschaft. Das beginnt bereits in den qualifizierenden Schulen, die mehr junge Menschen mit der Hochschulreife ausstatten, als das vor Jahren der Fall war. Zu dieser Gruppe zählt eine ebenfalls wachsende Zahl an Studierenden mit Migrationshintergrund. Unter dem Stichpunkt Diversity ist das Thema seit einigen Jahren in der politischen wie in der Hochschullandschaft auffallend präsent.

Vor diesem Hintergrund hat das Thema Lerncoaching für Hochschulen deutlich an Bedeutung gewonnen. Die Fachhochschule Südwestfalen hat die skizzierte Situation ernst genommen und sogenannte Studierendencoaches an allen vier Standorten etabliert. Das Besondere ist die Einordnung im Organigramm. Die Studierendencoaches sind als wissenschaftliche Mitarbeiter/-innen den Dekanaten der Fachbereiche zugeordnet und somit Teil des wissenschaftlichen Kollegiums. Das hat enorme Vorteile, weil Aktivitäten mit den Lehrenden koordiniert werden können, die Informationswege kurz sind und Dozenten Studierende mit Lernoder persönlichen Problemen direkt an den Studierendencoach verweisen können. Das Team der Studierendencoaches steht trotz der räumlichen Distanz der Standorte in einem engen Kontakt, so dass grundlegende Konzepte für alle Studierende der Hochschule zur Verfügung stehen. Im Detail hat jeder Studierendencoach ihre bzw. seine persönliche Handschrift: Individuelle Studienplanung, Lerncoaching, Interventionen gegen Prüfungsstress und psychosoziales Coaching sind die wesentlichen Handlungsfelder des Teams. Da ich der verantwortliche Coach am Standort Meschede bin, berichte ich im Folgenden überwiegend aus dieser Perspektive. Selbstverständlich studieren bei uns auch sehr gute bis exzellente Studierende. In diesem Beitrag richtet sich aufgrund des Themas der Fokus aller- 
dings auf die Studierenden mit Motivations- oder Lernschwierigkeiten - die sich später durchaus zu sehr guten Absolventen entwickeln können, wenn sie ihre Lernstrategie gefunden haben.

Der Studienerfolg hängt neben den kognitiven Möglichkeiten eines Menschen im Wesentlichen von seiner Motivation ab. Meiner Beobachtung nach haben wir es vermehrt mit Studierenden zu tun, die nur unzureichend intrinsisch motiviert sind und kein echtes Interesse an den fachlichen Inhalten zeigen. Vielmehr sehen diese Studierenden das Studium als reines Mittel zum Zweck: Der Bachelor- und ggf. Masterabschluss soll den persönlichen beruflichen Aufstieg sichern; der akademische Abschluss wird als Eintrittskarte in den Job gesehen. Diese Perspektive ändert sich bei vielen Studierenden erst zum Abschluss, wenn sie mit den Anforderungen der potenziellen Arbeitgeber konfrontiert werden.

Die Begleitung der Studierenden geschieht an den vier Standorten in allen Studienphasen. Der persönliche Kontakt ist uns wichtig, so dass uns bereits die Studienanfänger persönlich kennen lernen. In Meschede organisiere ich den Rahmen des Vorkurses Mathematik und begrüße die Teilnehmer am ersten Tag. Hier biete ich flankierend zur Mathematik Info-Veranstaltungen an, die ich z. T. selbst durchführe. Das Herzstück heißt Studienstrategien. Ich zeige den Übergang von der Schule zur Hochschule auf und lege den Fokus auf die Aspekte, die im Studium deutlich anspruchsvoller sind. Bereits in dieser Veranstaltung zeigt sich, dass die meisten Studienanfänger kaum über bewusste Lernstrategien verfügen. Zwar hat sich das Engagement in der Vermittlung von Lernstrategien an Schulen in den letzten Jahren deutlich erhöht, doch scheinen die Schüler/-innen diese Methodenkompetenzen primär als Lerninhalte zu verstehen, die nicht konsequent genutzt werden. Allerdings fehlt zu Studienbeginn im Allgemeinen eine Einsicht dafür, dieses Defizit auszugleichen.

Daher bieten wir ein breites Spektrum an unterstützenden Programmen an. Hierzu zählt zunächst die individuelle Studienplanung, die wir Studium flexibel nennen. Einem großen Anteil der Studienanfänger/-innen gelingt der Übergang von der Schule zur Hochschule nicht gut. Studienanfänger unterschätzen den erhöhten Workload. Getreu dem Motto „Früher lief es auch immer mit geringem Lernaufwand" bereitet sich dieser erschreckend hohe Anteil zu spät auf die Prüfungen vor. In dieser verspäteten Vorbereitungsphase wird den Studierenden bewusst, dass sie das Pensum nicht mehr schaffen und melden sich vor der Prüfung wieder $\mathrm{ab}$ oder provozieren sehenden Auges einen Fehlversuch. Auf diese Weise agieren viele in den folgenden Semestern weiter, gehen lediglich vermeintlich leichte Prüfungen an, "schieben“ die für sie anspruchsvollen und landen vielfach erst in höheren Semestern bei mir in der
Beratung. In dieser Situation verzweifeln diese Studierende und fürchten, der Situation nicht mehr Herr zu werden. Lernen ist eine individuelle Angelegenheit. Studierende orientieren sich oft zu stark an den Regelplänen, am Tempo von Kommilitonen oder anderen äußeren Faktoren. Letztlich können sie sich aber nur an sich selbst orientieren. Die Grundfrage sollte also nicht lauten: "Was sollte ich in diesem Semester schaffen?", sondern "Was kann ich in diesem Semester realistisch leisten?"

Je nach den individuellen Vorgaben der Prüfungsordnungen haben Studierende die Möglichkeit, das Tempo selbst $\mathrm{zu}$ bestimmen. Regelverlaufspläne sind Konstrukte, wie man das Studium aufteilen könnte. Sie sind aber nicht in Stein gemeißelt. Am Standort Meschede schwören weder Dozenten noch Studierendencoaches die Studierenden auf die Regelstudienzeit ein; diesem Druck unterwerfen sich vor allem die Studienanfänger selbst.

In Meschede beginnt das Programm Studium flexibel in der Regel nach vier Wochen. In den ersten vier Wochen studieren alle „nach Plan“ und testen, ob der volle Workload von 30 Credit Points funktioniert. In einer Info-Veranstaltung erfahren die Studierenden Details zum Programm. Für Studium flexibel können verschiedene Gründe sprechen:

- Grundlegende Kompetenzen müssen zunächst erarbeitet werden (z. B. Mathematik),

- das hohe Vermittlungstempo und die hohe Stoffdichte überfordern die Person,

- Studierende wollen/müssen nebenbei einem Nebenjob nachgehen oder

- Studierende sind bereits Eltern oder pflegen Angehörige.

Je nach individueller Situation und Leistungsfähigkeit definieren wir im gemeinsamen Gespräch einen realistischen Plan für das erste Semester. Mindestens einmal im Semester kommen die Studierenden zu Feedbackgesprächen, um den Plan anzupassen oder weitere Maßnahmen zu starten, wie sie im Folgenden erläutert werden. Ändert sich die individuelle Situation oder kommen Studierende nach einiger Zeit besser zurecht, kann der Workload entsprechend erhöht werden. Auch $\boldsymbol{B} \boldsymbol{A}$ föG-Empfänger können flexibel studieren, da der Leistungsnachweis zum 5. Fachsemester moderat definiert ist und das Programm berücksichtigt. Den Teilnehmern wird allerdings vermittelt, dass sie dennoch Leistungen nach $B A f o ̈ G$ immer nur für die Regelstudienzeit erhalten können. Anschließend greifen Konzepte für Werkstudententätigkeiten in Unternehmen.

Das Programm ist solide und funktioniert nachweislich gut, die Studierenden lernen, sich in ihrem Leistungsvermögen einzuschätzen und nicht zu überfordern. Teilnehmer des Programms sind anschließend stolz auf sich, weil sie nach ihren individuellen Möglichkeiten teilweise sehr gute Prüfungsergebnisse erzielt haben, während andere Kommilito- 
nen vermeintlich glaubten, dass es schon „irgendwie liefe“ und lediglich Fehlleistungen verbuchen konnten. Das ist auch das Kernproblem am Programm: Zu Beginn ihres Studiums glauben die meisten Studienanfänger, dass alles klappen würde und sie in Regelstudienzeit das Studium erfolgreich abschlössen, gute Noten erhielten und außerdem nebenbei arbeiten könnten. Diese Hoffnung erweist sich für einen recht großen Teil der Studierenden als Illusion. Daher biete ich Studium flexibel nicht ausschließlich $\mathrm{zu}$ Studienbeginn an, sondern in allen Phasen des Studiums. Kommen Studierende erst in hohen Semestern zu mir, ist ihnen immer noch zu helfen, aber die Studiendauer erhöht sich deutlicher, als wären sie frühzeitig aktiv geworden. Erfreulicherweise finden einige Studienanfänger/-innen noch vor dem Vorlesungsbeginn zu mir und starten direkt mit dem individuellen Plan in das erste Semester.

Mit dem strukturierten Rahmen von Studium flexibel ist eine Grundlage geschaffen, jedoch genügt dies für ein erfolgreiches Studium nicht zwingend. Das Lerncoaching hat sich in den letzten Jahren zu einem Programm für alle Studierende entwickelt, die ihr Lernmanagement entwickeln oder optimieren möchten.

Idealerweise beginnt die Arbeit zu Semesterbeginn, um den passenden Workload zu planen. Hierzu gehört auch die Auswahl von eventuell nachzuholenden und neuen Modulprüfungen. Da vor allem Studierende mit hohen Semesterzahlen dazu neigen, sich zu übernehmen, weil sie endlich ihr Studium abschließen wollen, ist es entscheidend, ein realistisches "Paket zu schnüren“. Als Grundlage fungiert mein umfangreiches Skript "Lern- und Arbeitsmethodik $^{\prime \prime}$, das in meinem Downloadbereich verfügbar ist: Gehirngerechtes Lernen, Zeit- und Selbstmanagement sowie Lese- und Visualisierungstechniken bildeten den Inhalt. Erkenntnisse aus der Lernforschung, Publikationen zum Themengebiet "Lernen lernen“ und vor allem persönliche Erfahrungen aus dem Lerncoaching haben das Skript geprägt; es ist speziell auf die Situation am Campus Meschede ausgerichtet. Hierauf aufbauend passen wir im Coaching Lernstrategien an, reflektieren den Lernprozess, optimieren Lerntechniken und - ganz wichtig - besprechen den Lernfortschritt. Diese Lernstandskontrolle ist für das Selbstbewusstsein der Studierenden entscheidend, weil sie sich und ihre Kompetenz vorher kaum realistisch einschätzen konnten. In aller Regel erhalten die Studierenden die notwendige Zuversicht, um ohne einschränkende Stresssymptome in die Klausur zu gehen.

Das gilt auch für die sensiblen Fälle vor dem letzten Prüfungsversuch. Am Standort Meschede haben Studierende in allen Studiengängen drei Prüfungsversuche ohne die Möglichkeit einer mündlichen Ergänzungsprüfung. Da mit der Zeit die Zahl der „Drittversuchler“, die Hilfe suchten, deutlich gestiegen ist, habe ich vor einigen Jahren das soge- nannte Drittversuchscoaching als festes Programm und ebenfalls zu einer "Marke” entwickelt. Es hat sich zu einem Erfolgsmodell mit einer ständig wachsenden Nachfrage gemausert, denn die Bestehensquote liegt bei ca. $98 \%$.

Üblicherweise prägen drei Handlungsweisen die defizitäre Lernstrategie:

- es mangelt an Struktur (Lernplanung),

- das Lernen beginn viel zu spät und

- die Coachees bereiten sich im Wesentlichen mit Übungsaufgaben und alten Klausuren vor, ohne zunächst die theoretischen Hintergründe $\mathrm{zu}$ erarbeiten - also ohne den Stoff zu verstehen.

Die interessierten Studierenden melden sich idealerweise zum Vorlesungsbeginn per E-Mail zum Programm an, indem sie mir eine formalisierte Bestandsaufnahme ihrer Situation zusenden. Im anschließenden Vorgespräch erörtern wir auf dieser Basis das individuelle Anliegen. In diesem Gespräch thematisiere Coach und Coachee das Empfinden von Prüfungsstress. Relativieren sich Stresssymptome nicht im Verlauf des Coachings, biete ich im Rahmen des psychosozialen Coachings eine Intervention zum Prüfungsstress an. Diese ressourcenorientierte Arbeit ist in aller Regel erfolgreich und die Studierenden lernen, in Prüfungen souverän und entspannt zu agieren. Da ich Autogenes Training als Entspannungsmethode vermittele, ergibt sich ein weiteres Element einer effizienten Arbeit bei der Bewältigung von Prüfungsstress.

Teil des Vorgesprächs sind Rahmenbedingungen für das Coaching. Die Coachees klären die fachliche Unterstützung durch Dozenten und/oder Mitarbeiter. Das funktioniert im Fachbereich sehr gut, da wir alle in einem intensiven Austausch stehen. In Einzelfällen kommt es zu gemeinsamen Termin mit dem Dozenten und mir, um speziellen Problemstellungen zu einer Lösung zu verhelfen.

Da die Coachees mitunter Schwierigkeiten haben, die neue Struktur durchzuhalten, ist die Absprache diesbezüglich sehr verbindlich. Die Coachees erarbeiten auf Basis meines Skripts einen Lernplan, der das gesamte Zeitmanagement für das Semester abbildet. Ferner verpflichten sich die Studierenden dazu, mich alle 14 Tage in einer von fünf möglichen offenen Sprechstunden in der Woche zu Feedbackgesprächen aufzusuchen.

Da bei uns vor allem die Wirtschafts- und Ingenieurmathematik ein Problemfeld darstellt, haben wir vor eineinhalb Jahren zusätzlich ein Mathematik-Lernlabor eingerichtet, das von zwei Mitarbeitern organisiert und im Wesentlichen durch Tutoren betreut wird. Das Lernlabor ist die gesamte Woche geöffnet und Studierende können spontan die Tutoren aufsuchen, um Defizite aufzuarbeiten oder auch dauerhaft betreut zu lernen. Dieses Angebot flankiert das Lern coaching in idealer Weise und wird von den Studierenden sehr gut angenommen. 
Üblicherweise hat sich bei den Coachees nach einigen Wochen die neue Lernstrategie etabliert, so dass die Feedbacktreffen ab der zweiten Semesterhälfte kürzer werden. Das allgemeine Lerncoaching und das Drittversuchscoaching laufen von der Struktur sehr ähnlich ab, nur das die Verbindlichkeit regelmäßiger Treffen im Drittversuchscoaching hinzukommt. Da die Feedbacktreffen im Rahmen meiner offenen Sprechstunden stattfinden und selten länger als 15 Minuten dauern, ist der Aufwand für die Coachees überschaubar und ich kann die stark gewachsene Nachfrage aktuell gut allein bewältigen. Über diese Treffen hinaus besitzen die Coachees die Möglichkeit, sich per E-Mail bei akuten Fragen außer der Reihe bei mir zu melden.

Die stets zunehmende Attraktivität des Programms ist gleichzeitig sein Problem: Zwar ist die Bestehensquote auf der Basis des Drittversuchscoachings seither erstaunlich hoch, doch es gelingt trotz aller prophylaktischen Maßnahmen wie den geschilderten Veranstaltungen zu Beginn des Studiums bislang kaum, Studienanfänger frühzeitig für das Thema Lernmanagement zu interessieren und es erst gar nicht zum Drittversuch kommen zu lassen. Nur ein geringer Teil der Erstsemester sucht bei Lemproblemen direkt die Studienberatung auf; vielmehr scheint es so, als müssten die meisten Studierenden erst selbst testen, ob "die Herdplatte wirklich heiß ist“. Das Gros der Hilfesuchenden befindet sich in höheren Fachsemestern.

Um Studienanfänger deutlicher zu motivieren, wurde gerade eine weitere Mitarbeiterstelle besetzt. Diese Position flankiert die Tätigkeit des Studierendencoaches durch Veranstaltungen zu Lern- und Methodenkompetenzen sowie weiteren Gruppenangeboten. Sie ist z. T. in der Peripherie zwischen dem Studierendencoaching und der Lehre angesiedelt (z. B. in Onboardingkonzepten im Rahmen von Lehrveranstaltungen im ersten Fachsemester), so dass sich die Kultur des Lerncoachings noch intensiver mit dem Studium verzahnen kann.

\section{Logistik im Überblick.}

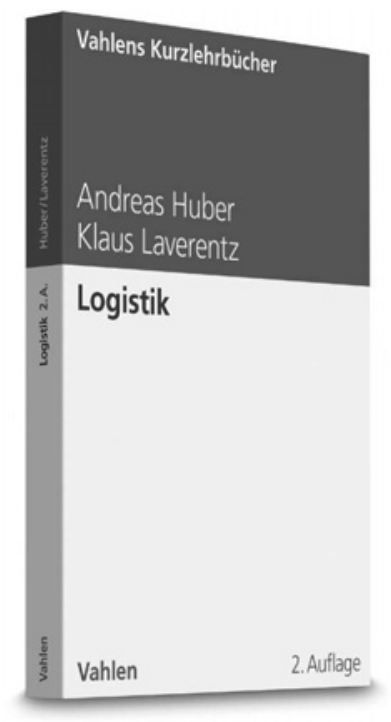

Huber/Laverentz Logistik

2. Auflage. 2019. XIV, 254 Seiten. Gebunden $€ 19,80$ ISBN 978-3-8006-5889-3

\section{Perfekter Einstieg}

Das Lehrbuch behandelt den klassischen Lehrstoff der Logistik und berücksichtigt gleichzeitig auch aktuelle Entwicklungen. Ziel des Buches ist es, den theoriebezogenen und praxisrelevanten Stoff der Logistik zu verzahnen.

\section{Umfassend}

Neben den klassischen Funktionen der Beschaffungslogistik, Produktionslogistik und Distributionslogistik stellt das Buch ein modernes Dienstleistungsverständnis von Logistik dar. Integriert werden insbesondere Supply Chain Management, logistische Kommunikations- und Informationssysteme, Qualitätsmanagement sowie Lager- und Transportmanagement.

\section{Vahlen}

